\title{
RANDOM FIXED POINTS OF NON-SELF MAPS AND RANDOM APPROXIMATIONS ${ }^{1}$
}

\author{
ISMAT BEG \\ Kuwait University, Department of Mathematics and Computer Science \\ P.O. Box 5969, Safat 13060, Kuwait \\ E-mail: IBEG@MATH-1.SCI.KUNIV.EDU.KW
}

(Received June, 1996; Revised December, 1996)

In this paper we prove random fixed point theorems in reflexive Banach spaces for nonexpansive random operators satisfying inward or LeraySchauder condition and establish a random approximation theorem.

Key words: Random Fixed Point, Nonexpansive Random Operator, Weak Inward Condition, Leray-Schauder Condition.

AMS subject classifications: $47 \mathrm{H} 10,60 \mathrm{H} 25,41 \mathrm{~A} 50$.

\section{Introduction}

Lin [6] proved a random version of an approximation theorem of Fan [3] and obtained several random fixed point theorems. Recently $\mathrm{Xu}$ [12] and Lin [7] obtained some more random fixed point theorems for self and non-self nonexpansive or condensing random operators. For other related work we refer the reader to $[1,2,8,9,10,11$, 13]. In this paper we prove random fixed point theorems in reflexive Banach spaces for nonexpansive random operators, and generalize the results obtained by $\operatorname{Lin}[6,7]$ and $\mathrm{Xu}$ [11]. A random version of best approximation theorem of Fan [3] is also derived.

\section{Preliminaries}

Throughout this paper, $(\Omega, \Sigma)$ denotes a measurable space with $\Sigma$ a sigma algebra of subsets of $\Omega$. Let $(X, d)$ be a metric space, $2^{X}$ be family of all subsets of $X$, and $W K(X)$ be family of all nonempty weakly compact subsets of $X$. A mapping $F: \Omega \rightarrow 2^{X}$ is called measurable if for any open subset $C$ of $X, F^{-1}(C)=\{w \in \Omega$ : $F(w) \cap C \neq \emptyset\} \in \Sigma$. A mapping $\xi: \Omega \rightarrow X$ is said to be a measurable selector of a measurable mapping $F: \Omega \rightarrow 2^{X}$ if $\xi$ is measurable and for any $w \in \Omega, \xi(w) \in F(w)$. Let $M$ be a subset of $X$. A mapping $T: \Omega \times M \rightarrow X$ is called a random operator if for any

${ }^{1}$ This research partially supported by the Kuwait University research grant No. SM 119. 
$x \in M, T(\cdot, x)$ is measurable. A measurable mapping $\xi: \Omega \rightarrow M$ is called a random fixed point of a random operator $T: \Omega \times M \rightarrow X$ if for every $w \in \Omega, \xi(w)=T(w, \xi(w))$.

A mapping $T: M \rightarrow X$ is called $k$-set-Lipschitz $(k \geq 0)$ if $T$ is continuous and for any bounded subset $B$ of $M, \alpha(T(B)) \leq k \alpha(B)$, where $\alpha(B)=\inf \{e>0: B$ can be covered by a finite number of sets of diameter $\leq e\}$. The number $\alpha(B)$ is called the (set)-measure of noncompactness of $B$. A $k$-set-Lipschitz mapping $T$ is a $k$-set-contraction if $k<1$. A mapping $T: M \rightarrow X$ is called (set-) condensing if $T$ is continuous and for each bounded subset $C$ of $M$ with $\alpha(C)>0, \alpha(T(C))<\alpha(C)$. Clearly a $k$ set-contraction mapping is condensing. A mapping $T: M \rightarrow X$ is called nonexpansive if $\|T(x)-T(y)\| \leq\|x-y\|$ for all $x, y \in M$. A random operator $T: \Omega \times M \rightarrow X$ is continuous (condensing, nonexpansive, etc.) if for each $w \in \Omega, T(w, \cdot)$ is continuous (condensing, nonexpansive, etc.) A random operator $T: \Omega \times M \rightarrow X$ is said to be weakly inward if for each $w \in \Omega, T(w, x) \in \operatorname{cl} I_{M}(x)$ for $x \in M$, where cl denotes closure and $I_{M}(x)=\{z \in X: z=x+a(y-x)$ for some $y \in M$ and $a \geq 0\}$. When $M$ has a nonempty interior, a random operator $T: \Omega \times M \rightarrow X$ is said to satisfy the LeraySchauder condition if for each $w \in \Omega$, there exists an element $z \in \operatorname{int}(M)$ (depending on $w$ ) such that

$$
T(w, y)-z \neq a(y-z)
$$

for all $y$ in the boundary of $M$ and $a>1$.

A mapping $T: M \rightarrow X$ is said to be demiclosed at $y \in X$ if, for any sequence $\left\{x_{n}\right\}$ in $M$, the conditions $x_{n} \rightarrow x \in M$ weakly and $T\left(x_{n}\right) \rightarrow y$ strongly imply $T(x)=y$.

Theorem 2.1: [Xu, 12]. Let $C$ be a nonempty closed convex subset of a separable Banach space $X, T: \Omega \times C \rightarrow X$ be a condensing random operator that is either (i) weakly inward or (ii) satisfies the Leray-Schauder condition. Suppose, for each $w \in \Omega, T(w, C)$ is bounded. Then $T$ has a random fixed point.

Remark 2.2: Theorem 2.1 remains true if $C$ is separable instead of $X$ being separable.

\section{The Main Results}

Theorem 3.1: Let $C$ be a nonempty closed bounded convex separable subset of a reflexive Banach space $X$ and let $T: \Omega \times C \rightarrow X$ be a weakly inward nonexpansive random operator. Suppose for each $w \in \Omega, I-T(w, \cdot)$ is demiclosed at zero. Then $T$ has a random fixed point.

Proof: Take an element $v \in C$ and a sequence $\left\{k_{n}\right\}$ of real numbers such that $0<k_{n}<1$ and $k_{n} \rightarrow 0$ as $n \rightarrow \propto$. For each $n$, define a mapping $f_{n}: \Omega \times C \rightarrow X$ by $f_{n}(w, x)=k_{n} v+\left(1-k_{n}\right) T(w, x)$. Then, $f_{n}$ is a weakly inward $\left(1-k_{n}\right)$-set-contraction random operator. Hence by Theorem $2.1(i)$ and Remark 2.2, there is a random fixed point $\xi_{n}$ of $f_{n}$. Since $X$ is a reflexive Banach space, $w-\operatorname{cl}\left\{\xi_{i}(w)\right\}$ is weakly compact.

Let $C$ be a weakly closed and bounded subset of $X$ containing $w-\operatorname{cl}\left\{\xi_{i}(w)\right\}$. For each $n$, define $F_{n}: \Omega \rightarrow W K(C)$ by $F_{n}(w)=w-\operatorname{cl}\left\{\xi_{i}(w): i \geq n\right\}$. Let $F: \Omega \rightarrow W K(C)$ be a mapping defined by $F(w)=\bigcap_{n=1}^{\infty} F_{n}(w)$. Then, as in Itoh [5, proof of Theorem $2.5], F$ is $w$-measurable and has a measurable selector $\xi$. This $\xi$ is the desired random fixed point of $T$. Indeed, fix any $w \in \Omega$, then some subsequence $\left\{\xi_{m}(w)\right\}$ of 
$\left\{\xi_{n}(w)\right\}$ converges weakly to $\xi(w)$. On the other hand, we have $\xi_{m}(w)-$ $T\left(w, \xi_{m}(w)\right)=k_{m}\left\{v-T\left(w, \xi_{m}(w)\right)\right\}$. Thus $\left\{\xi_{m}(w)-T\left(w, \xi_{m}(w)\right)\right\}$ converges to 0 . Since $I-T(w, \cdot)$ is demiclosed at zero, it follows that $\xi(w)=T(w, \xi(w))$.

If $T: \Omega \times C \rightarrow C$ then we have the following:

Theorem 3.2: Let $C$ be a nonempty closed bounded convex separable subset of a reflexive Banach space and let $T: \Omega \times C \rightarrow C$ be a nonexpansive random operator. Suppose for each $w \in \Omega, I-T(w, \cdot)$ is demiclosed at zero. Then $T$ has a random fixed point.

Theorem 3.3: Let $C$ be a nonempty closed bounded convex separable subset of a reflexive Banach space $X$ and has a nonempty interior. Let $T: \Omega \times C \rightarrow X$ be a nonexpansive random operator that satisfies the Leray-Schauder condition. Suppose for each $w \in \Omega, I-T(w, \cdot)$ is demiclosed at zero. Then $T$ has a random fixed point.

Proof: Let $z=z(w) \in \operatorname{int}(C)$ satisfy inequality (1). Take a sequence $\left\{k_{n}\right\}$ of real numbers such that $0<k_{n}<1$ and $k_{n} \rightarrow 0$ as $n \rightarrow \infty$. For each $n$, define a mapping $f_{n}$ : $\Omega \times C \rightarrow X$ by $f_{n}(w, x)=k_{n} z+\left(1-k_{n}\right) T(w, x)$. Then $f_{n}$ is a random $\left(1-k_{n}\right)$-set-contraction operator that satisfies the Leray-Schauder condition. Then, by Theorem 2.1 (ii) and Remark $2.2, f_{n}$ has a random fixed point $\xi_{n}$. Define a sequence of mappings $F_{n}: \Omega \rightarrow W K(C)$ and a mapping $F: \Omega \rightarrow W K(C)$ as in the proof of Theorem 3.1. Then $F$ is measurable and has a measurable selector $\xi$. This $\xi$ is the desired random fixed point of $T$.

The following is a special case of Theorem 3.2, which extends the results of Lin [6, Theorem 3] and Lin [7, Corollary 3.2].

Theorem 3.4: Let $C$ be a nonempty closed bounded convex separable subset of a Hilbert space $X$ and let $T: \Omega \times C \rightarrow X$ be a nonexpansive random operator. Then there exists a measurable map $\xi: \Omega \rightarrow C$ such that

$$
\|\xi(w)-T(w, \xi(w))\|=d(T(w, \xi(w)), C)
$$

for each $w \in \Omega$.

Proof: Let $P$ be the proximity map on $C$, that is, $P$ is a continuous map from $X$ into $C$ such that for each $y \in X$ we have

$$
\|P(y)-y\|=d(y, C)
$$

Since both $P$ and $T$ are nonexpansive, the random operator $P \circ T: \Omega \times C \rightarrow C$ is also nonexpansive. By Theorem 3.2 there exists a random fixed point of $P \circ T$, that is, there exists a measurable map $\xi: \Omega \rightarrow C$ such that $P \circ T(w, \xi(w))=\xi(w)$, for each $w \in \Omega$. Therefore,

for each $w \in \Omega$.

$$
\begin{gathered}
\|\xi(w)-T(w, \xi(w))\|=\|P \circ T(w, \xi(w))-T(w, \xi(w))\| \\
=d(T(w, \xi(w)), C)
\end{gathered}
$$

\section{Remark 3.5:}

(i) Immediate corollaries to Theorems 3.1 are Lin $\left[6\right.$, Theorem $\left.6^{\prime}(i i)\right]$ and Lin $[7$, Corollary 4.2 (iii)].

(ii) Theorem 3.2 generalizes Lin [6, Lemma 1] and $\mathrm{Xu}[12$, Theorem 1].

(iii) The fixed point property of $C$ and strict convexity of $X$ in Xu [12, Theorem 1] are not needed. 
(iv) Theorem 3.3 extends $\mathrm{Xu}[12$, Theorem 4].

\section{References}

[1] Beg, I. and Shahzad, N., Random fixed points of random multivalued operators on Polish spaces, Nonlinear Analysis 20 (1993), 835-847.

[2] Beg, I. and Shahzad, N., Random fixed points of weakly inward operators in conical shells, J. Appl. Math. Stoch. Anal. 8 (1995), 261-264.

[3] Fan, Ky, Extensions of two fixed point theorems of F.E. Browder, Math. Z. 112 (1969), 234-240.

[4] Himmelbeg, C.J., Measurable relations, Fund. Math. 87 (1975), 53-72.

[5] Itoh, S., Random fixed point theorems with an application to random differential equations in Banach spaces, J. Math. Anal. Appl. 67 (1979), 261-273.

[6] Lin, T.C., Random approximations and random fixed point theorems for nonself maps, Proc. Amer. Math. Soc. 103 (1988), 1129-1135.

[7] Lin, T.C., Random approximations and random fixed point theorems for continuous 1-set contractive random maps, Proc. Amer. Math. Soc. 123 (1995), 1167-1176.

[8] Papageorgiou, N.S., Random fixed point theorems for multifunctions, Math. Japonica 29 (1984), 93-106.

[9] Sehgal, V.M. and Waters, C., Some random fixed point theorems, Contemporary Math. 21 (1983), 215-218.

[10] Tan, K.K. and Yuan, X.Z., Some random fixed point theorems, Fixed Point Theory and Applications (ed. by K.K. Tan), World Scientific, Singapore (1992), 334-345.

[11] Wagner, D.H., Survey of measurable selection theorems, SIAM J. Contr. Optim. 15 (1977), 859-903.

[12] Xu, H.K., Some random fixed point theorems for condensing and nonexpansive operators, Proc. Amer. Math. Soc. 110 (1990), 395-400.

[13] Yuan, X.Z. and Roy, J.M., Some deterministic and random variational inequalities and their applications, J. Appl. Math. and Stoch. Anal. 8 (1995), 381-395. 


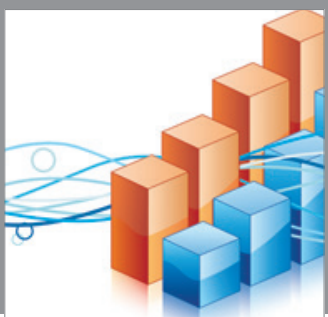

Advances in

Operations Research

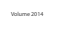

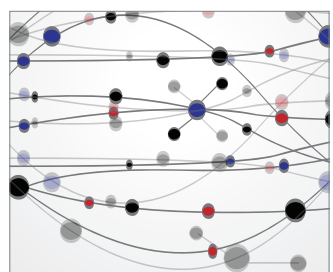

\section{The Scientific} World Journal
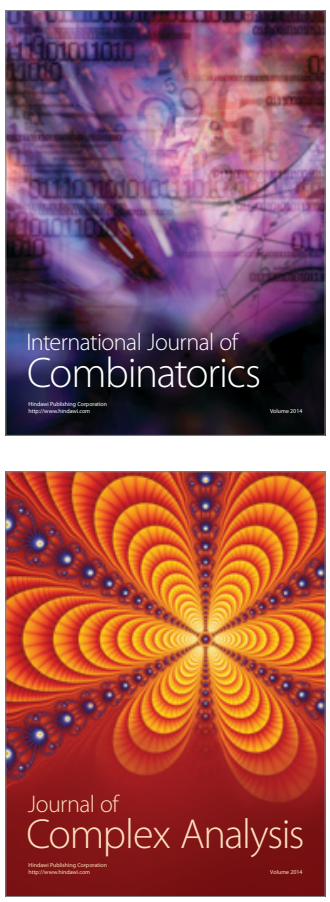

International Journal of

Mathematics and

Mathematical

Sciences
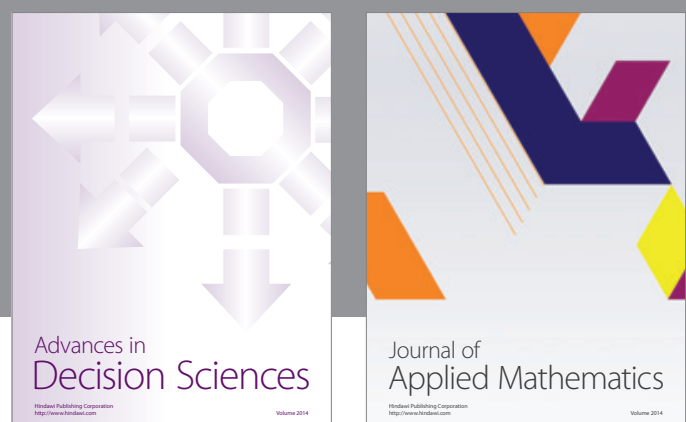

Journal of

Applied Mathematics
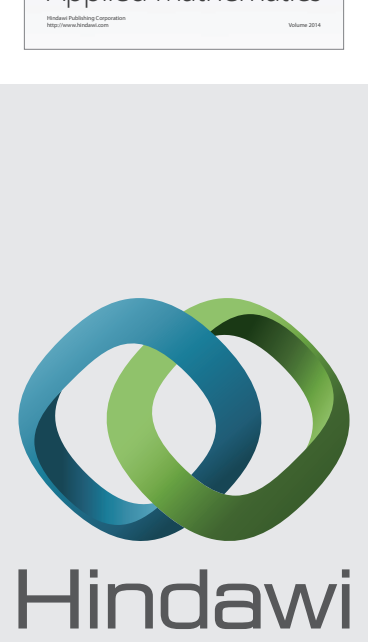

Submit your manuscripts at http://www.hindawi.com
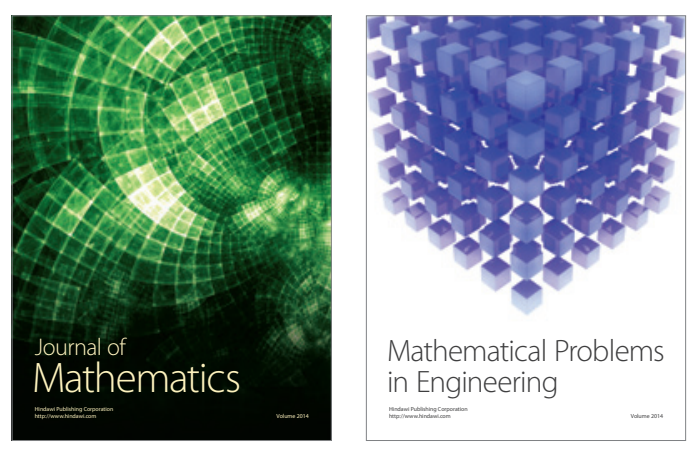

Mathematical Problems in Engineering
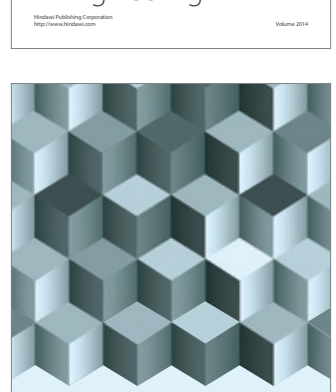

Journal of

Function Spaces
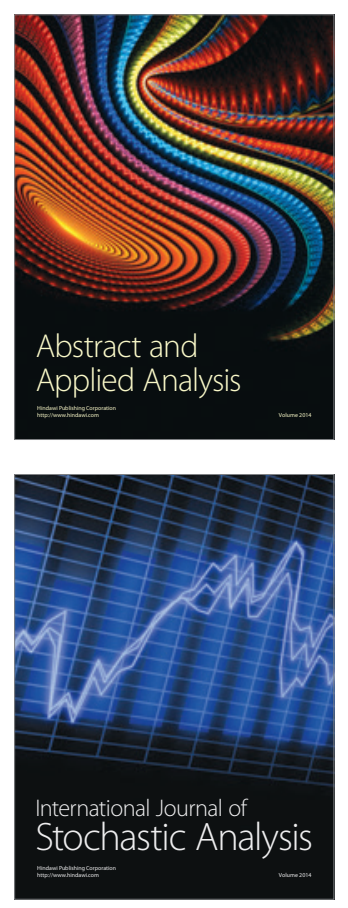

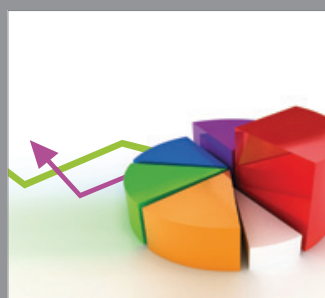

ournal of

Probability and Statistics

Promensencen
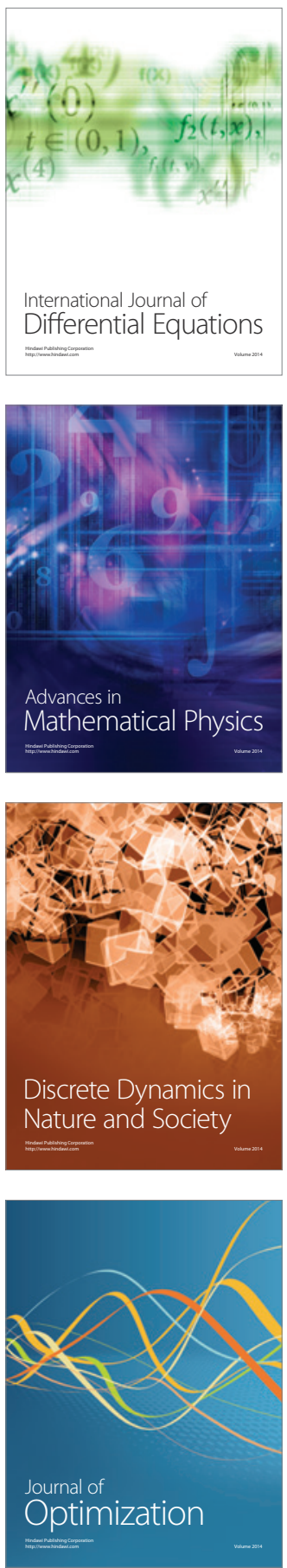